\title{
Effect of an adiabatic fin on natural convection heat transfer in a triangular enclosure
}

\author{
Sreebash C Paul ${ }^{1}$, Suvash C. Saha ${ }^{2,}$, Y. T. Gu ${ }^{2}$ \\ ${ }^{1}$ Department of Arts and Sciences, Ahsanullah University of Science and Technology (AUST), Dhaka 1208, Bangladesh \\ ${ }^{2}$ School of Chemistry, Physics \& Mechanical Engineering, Queensland University of Technology, Brisbane, QLD 4001 , Australia
}

\section{Email address:}

s_c_saha@yahoo.com(S. C. Saha), suvash.saha@qut.edu.au(S. C. Saha)

\section{To cite this article:}

Sreebash C Paul, Suvash C. Saha, Y. T. Gu. Effect of an Adiabatic Fin on Natural Convection Heat Transfer in a Triangular Enclosure. American Journal of Applied Mathematics. Vol. 1, No. 4, 2013, pp. 78-83. doi: 10.11648/j.ajam.20130104.16

\begin{abstract}
Natural convection thermal boundary layer adjacent to the heated inclined wall of a right angled triangle with an adiabatic fin attached to that surface is investigated by numerical simulations. The finite volume based unsteady numerical model is adopted for the simulation. It is revealed from the numerical results that the development of the boundary layer along the inclined surface is characterized by three distinct stages, i.e. a start-up stage, a transitional stage and a steady stage. These three stages can be clearly identified from the numerical simulations. Moreover, in presence of adiabatic fin, the thermal boundary layer adjacent to the inclined wall breaks initially. However, it is reattached with the downstream boundary layer next to the fin. More attention has been given to the boundary layer development near the fin area.
\end{abstract}

Keywords: Heat Transfer, Triangular Enclosure, Fin, Boundary Layer

\section{Introduction}

Heat transfer due to natural convection in triangular enclosures is of fundamental interest in the fluid mechanics research community and has application to buildings, solar collectors, and greenhouses. Controlling heat transfer by improving or depressing natural convection flows within an enclosure is of considerable importance in industrial applications. The use of fins on the wall of an enclosure is an efficient way to alter heat transfer through the cavity.

A study on heat transfer by free convection in right triangular enclosures was performed by Akinsete and Coleman, 1982. The base, the inclined wall and the vertical wall of the enclosure was assumed to be cooled, heated and adiabatic respectively. In their investigation it was found an increase in heat transfer near the apex of the cooled and heated wall. To investigate this issue, Moukalled and Acharya, 2000, 2001 investigated natural convection in a trapezoidal enclosure by mounting baffles from the base and the inclined wall, and they observed a significant decrease in heat transfer.

$\mathrm{Xu}$ et al., 2008, 2009 and 2010 performed experimental well as numerical investigation on natural convection in a differentially heated rectangular cavity with or without fin on a sidewall for high Rayleigh number. They observed strong oscillations in the thermal flow above the fin caused by a Rayleigh-Benard-type instability in the flow. Oscillations in the boundary layer triggered the boundary layer into transition to turbulence, which in turn enhances the total heat transfer through the fined sidewall.

Varol et al., 2007 investigated the effect of thin fin on natural convection in triangular enclosures. The enclosure was filled with porous media and an adiabatic solid vertical thin fin was placed on the bottom wall. It is observed that the thin fin can be a passive control parameter for flow field, temperature distribution and heat transfer. They reported that the addition of fin enhances heat transfer and the value of the mean Nusselt number decreases by increasing dimensionless fin height. Ridouane and Campo, 2007 also reduced the heat transfer by attaching baffles to the inclined walls of an isosceles triangular shaped enclosure.

The effect of thermally conducted fin on natural convection in triangular enclosure filled with porous media is again investigated by Varol et al., 2008. They showed that thermal conductivity of the fin attached horizontally to the vertical wall does not have any significant effect on natural convection, however, presence of fin does change the flow fields and temperature distributions. It was also observed that the heat transfer decreases with the increasing fin length as well as distance of the fin position.

Recently, Anderson et al., 2009 used an adiabatic baffle 
mounted vertically downward from the apex of an attic shaped enclosure. One of the inclined walls was considered as heated while the other inclined wall and base were assumed as cold. In their study, it was found that the use of a single adiabatic baffle mounted vertically downward from the apex would alter the flow such that the convective heat loses were suppressed. It was also observed that the convective heat transfer was reduced as the length of the baffle was increased. Most recently, Anderson et al., 2010 investigated experimentally by using thick polystyrene baffle; firstly mounting from the apex of the enclosure downwards and secondly from the centre of the base upwards. From their study, it was also found that increasing baffle length mounted, either from the apex down or the base up, changes the flow pattern which in turn suppress the heat transfer by the natural convection.

In this study, convective thermal boundary layer adjacent to the heated inclined wall of a right angled triangle with an adiabatic fin mounted from that wall is investigated by numerical simulations. The governing N-S and the energy equations are solved numerically for different Rayleigh number, $R a$, in the range of $10^{5}-10^{8}$ keeping aspect ratio, $A=$ 1.0, and Prandtl number, $\operatorname{Pr}=0.71$, fixed. The development of the boundary layer along the inclined heated wall is investigated. The effect of $R a$ on the heat transfer is also investigated. More attention has been given to the boundary layer development near the fin area.

\section{Problem Formulations}

The physical system is sketched in Fig. 1, which is an air filled right angled triangular enclosure. $\mathrm{H}$ is the height of the enclosure and $\mathrm{L}$ is the length of the base. Tc and Th are the cold and hot temperature applied to the base and inclined wall respectively.

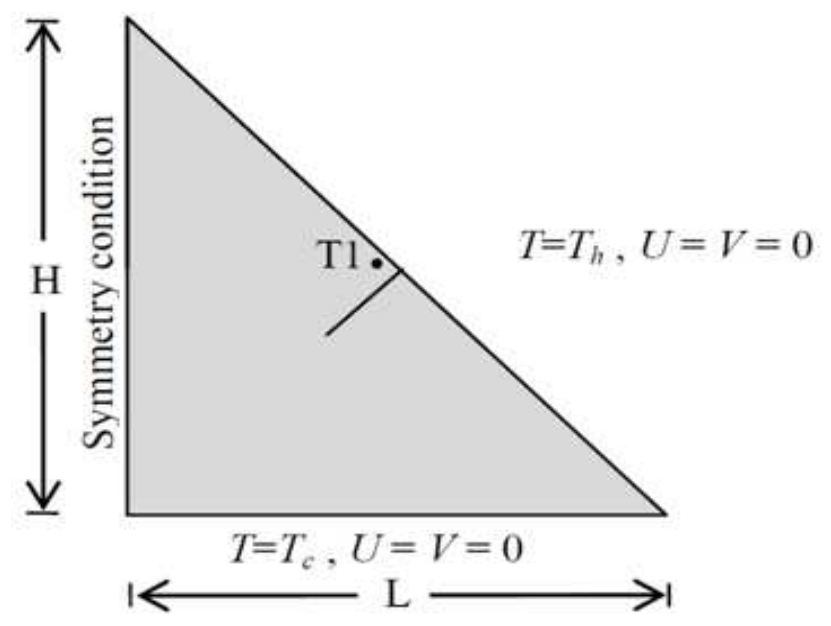

Fig 1. A schematic of the geometry and boundary conditions

The development of natural convection inside a triangular enclosure is governed by the following non-dimensional Navier-Stokes and energy equations with the Boussinesq approximation:

$$
\begin{gathered}
\frac{\partial u}{\partial x}+\frac{\partial v}{\partial y}=0 \\
\frac{\partial u}{\partial \tau}+u \frac{\partial u}{\partial x}+v \frac{\partial u}{\partial y}=-\frac{\partial p}{\partial x}+\operatorname{Pr}\left(\frac{\partial^{2} u}{\partial x^{2}}+\frac{\partial^{2} u}{\partial y^{2}}\right) \\
\frac{\partial v}{\partial \tau}+u \frac{\partial v}{\partial x}+v \frac{\partial v}{\partial y}=-\frac{\partial p}{\partial x}+\operatorname{Pr}\left(\frac{\partial^{2} v}{\partial x^{2}}+\frac{\partial^{2} v}{\partial y^{2}}\right)+\operatorname{Pr} \operatorname{Ra} \theta \\
\frac{\partial \theta}{\partial \tau}+u \frac{\partial \theta}{\partial x}+v \frac{\partial \theta}{\partial y}=\left(\frac{\partial^{2} \theta}{\partial x^{2}}+\frac{\partial^{2} \theta}{\partial y^{2}}\right)
\end{gathered}
$$

where $\mathrm{x}, \mathrm{y}, \mathrm{u}, \mathrm{v}, \theta, \mathrm{p}$ and $\tau$ are non-dimensional horizontal and vertical axes, horizontal and vertical velocity components, temperature, pressure and time respectively. The above non-dimensional variables are obtained by normalizing their dimensional values with the following expressions.

$$
\begin{aligned}
& x=\frac{X}{H}, y=\frac{Y}{H}, u=\frac{U}{\kappa / H}, v=\frac{V}{\kappa / H}, \tau=\frac{t}{H^{2} / \kappa} \\
& p=\frac{P}{\rho \kappa^{2} / H^{2}}, \theta=\frac{T-T_{c}}{T_{h}-T_{c}}
\end{aligned}
$$

Here, the three dimensionless parameters which govern the natural convection flow are the Rayleigh number $(R a)$, the Prandtl number $(P r)$ and the aspect ratio $(A)$ defined as

$$
R a=\frac{g \beta\left(T_{h}-T_{c}\right) H^{3}}{v \kappa}, \operatorname{Pr}=\frac{v}{\kappa}, A=\frac{H}{L}
$$

where $v, \rho, \beta, \kappa$ are the kinematic viscosity, density of the fluid, coefficient of thermal expansion and thermal diffusivity respectively, and $g$ is the acceleration due to gravity.

The dimensionless boundary conditions are as follows:

$$
\begin{gathered}
u=v=0, \theta=0 \quad \text { along the horizontal wall } \\
u=v=0, \theta=1 \quad \text { along the inclined wall } \\
\frac{\partial u}{\partial x}=\frac{\partial v}{\partial x}=\frac{\partial \theta}{\partial x}=0 \quad \text { along the vertical wall }
\end{gathered}
$$

The Nusselt number, $N u$, which is a physical quantity to measure heat transfer, is 


$$
N u=\frac{q H}{k \Delta T}
$$

where $q, k$ are the total convective heat flux and thermal conductivity respectively, and $\Delta T$ is the temperature difference between hot and cold wall.

\section{Numerical Scheme}

Equations (1)-(4) are solved along with the initial and boundary conditions using the SIMPLE scheme. The Finite Volume method has been chosen to discretize the governing equations, with the QUICK scheme approximating the advection term. The diffusion terms are discretized using central differencing with second order accuracy. A second order implicit time-marching scheme has also been used for the unsteady term. The detailed numerical procedure can be found in Saha, 2010.

To capture the thermal boundary layer adjacent to the heated inclined wall and the flow field around the fin, finer meshes are distributed near the inclined wall and around the fin compared to other regions. A total of 12367 grid nodes and time step of $1.59 \times 10^{-7}$ are considered for all computations. We have performed grid dependency test by halving the grid size and doubling the time steps. The reported errors were in the acceptable ranges $(<2 \%)$. The detailed tests and results are not presented here for brevity.
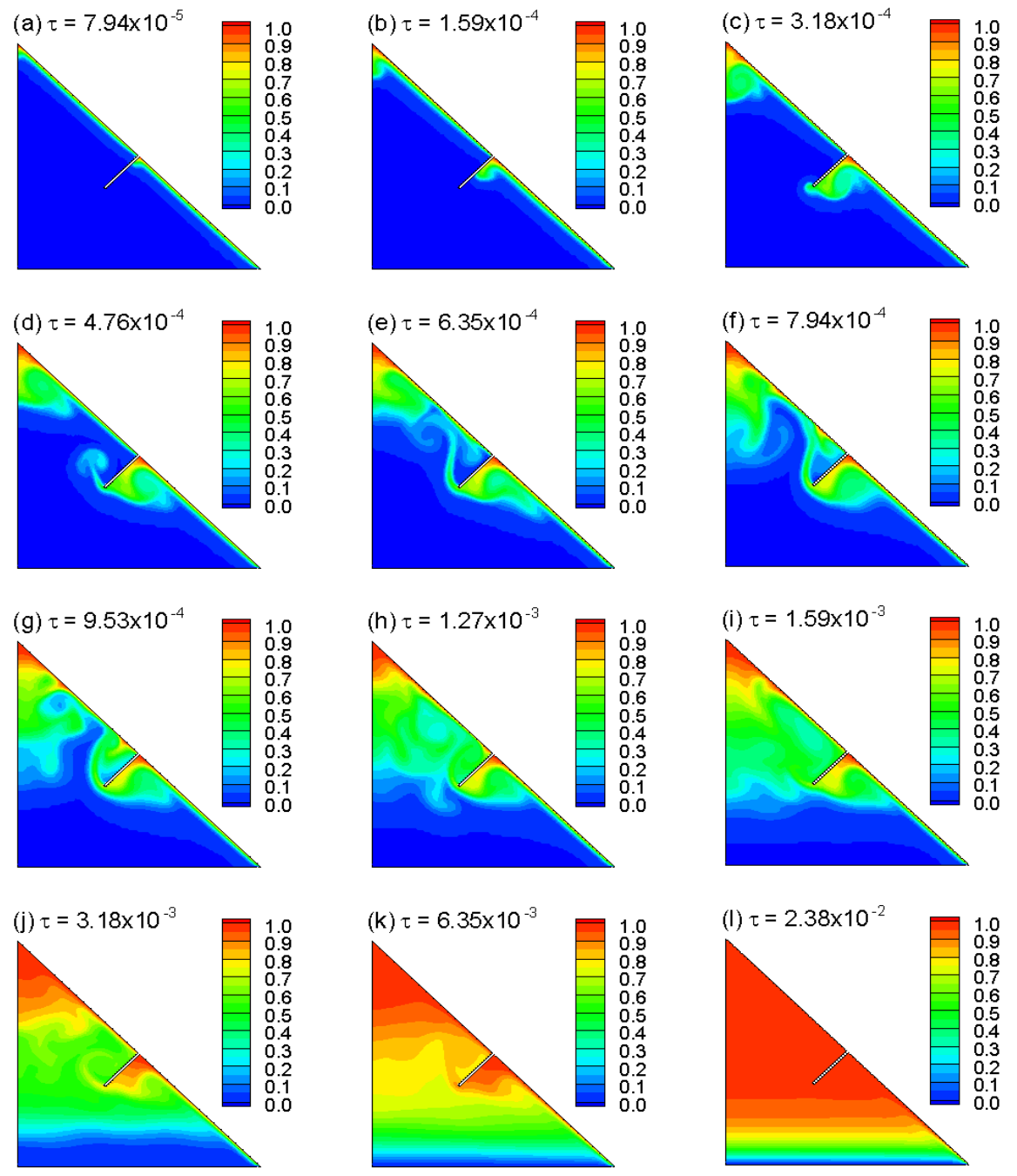

Fig 2. Temperature contours at different times for $\mathrm{Ra}=10^{8}$

\section{Results and Discussions}

In Fig. 2, contours of the temperature are presented at different times for $R a=10^{8}$. The figure shows that initially the boundary layer develops adjacent to the hot inclined walls of the enclosure. However, as time increases, the top of the enclosure fills gradually with hot fluid and becomes stratified, where the top portion fluid is hotter than the bottom portion.

The fin mounted from the middle on the inclined wall blocks the upstream thermal boundary layer flow and causes the heated fluid to accumulate underneath the fin. As a result, a lower intrusion is formed, as seen in Fig. 2(a). As time 
passes, the lower intrusion front moves along and toward the head of the fin due to the continuous accumulation of the heated fluid as seen in Fig. 2(a-c). As soon as the lower intrusion front arrives at the end of the fin, it starts to rise vertically due to buoyant force. Fig. 2(d-1) show the subsequent development of the thermal flow above the fin. It also demonstrates that, as the starting plume moves upwards, the plume becomes increasingly unstable and strikes the downstream thermal boundary layer next to the fin as observed in Fig. 2(e). The boundary layer then breaks. However, it is reattached as time passes as seen in Fig. 2(j).
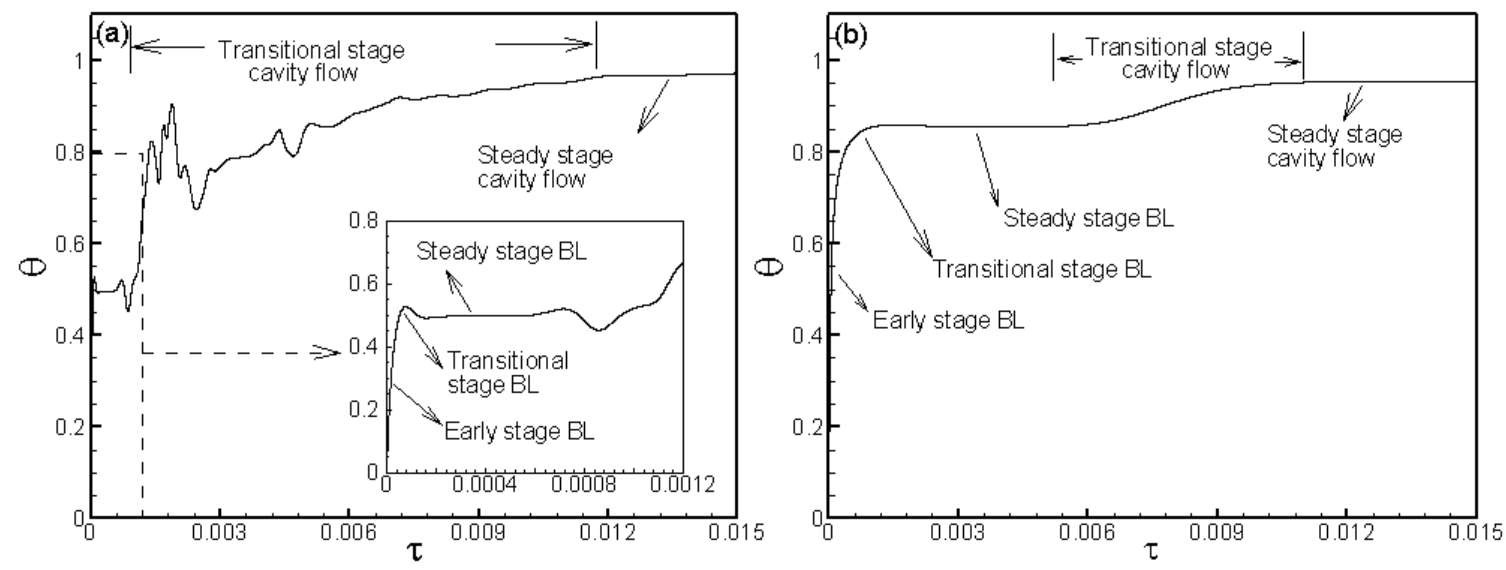

Fig 3. Time series of temperature in the downstream boundary layer at $T 1(0.46394,0.52667)$ (a) $R a=10^{8} \&(b) R a=10^{6}$.

The time series of temperature recorded at $\mathrm{T} 1(0.46394$, 0.52667 ) above the fin are shown in Fig. 3 for $R a=10^{8}$ and $10^{6}$. The figure shows that initially the temperature on this point is 0 which is the initial temperature of the enclosure. It is found from this figure that the air flow is dominated by two distinct stages of development, i.e. a boundary-layer development stage and the thermal stratification of the cavity. The thermal boundary layer along the inclined surface is characterized by three distinct stages, i.e. a start-up early stage, a transitional stage and a steady stage which has been shown in both Fig. 3(a) and (b). Then the stratification stage starts with a transitional stage followed by a steady stage of the entire enclosure. The early stage of the boundary layer development is dominated by conduction. The stage ceases when the conduction balances convection. The transitional stage is dominated by overshoot and undershoot. At the steady stage the conduction completely balances with convection. The thermal boundary layer, which develops adjacent to the inclined wall reaches the apex of the enclosure and starts to heat the top portion as a form of thermal stratification. This process continues until the whole cavity becomes steady state.

Fig. 4 shows the time series of average $N u$ which have been calculated from the hot inclined wall of the enclosure for different $R a$. The figure shows the dependency of $N u$ on $R a$. It is seen that initially the $N u$ is very high because of the conduction effect. As the time increases, it decreases gradually and become steady state when the whole enclosure becomes stratified. The three stages of boundary layer development can also be identified from here. For Ra $=10^{8}$, the Nusselt number oscillates during the boundary layer development stage due to strong buoyancy effect. However, as time progress the flow becomes stratified and the oscillation disappears.

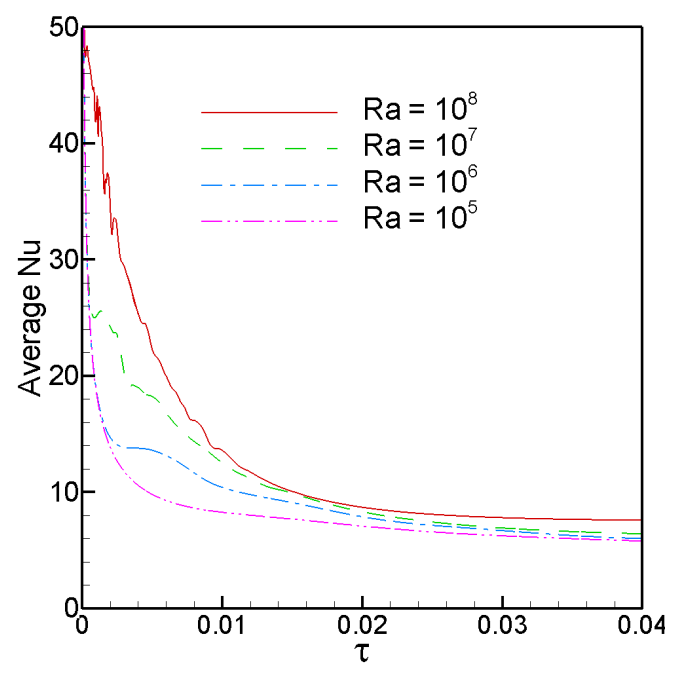

Fig 4. Time series of average Nu on the inclined surface of the enclosure for different $R a$

Surface $N u$ on the inclined heated wall of the enclosure for different $R a$ is presented in Fig. 5. The $N u$ is almost zero on the inclined wall above the fin. This is because, due to thermal stratification the top portion of the enclosure is almost heated up (interior temperature is equal to the surface temperature). Therefore, there is no heat transfer should be expected through that surface. However, bellow the fin the heat transfer increases gradually towards the bottom tip of the enclosure. Since the bottom tip connect the bottom cold surface and inclined heated surface, the heat transfer tends to infinity at the tip due to singularity. It is also noticed that higher values of $N u$ are obtained for higher values of $R a$ because the natural convection becomes dominant to convection due to higher $R a$. 


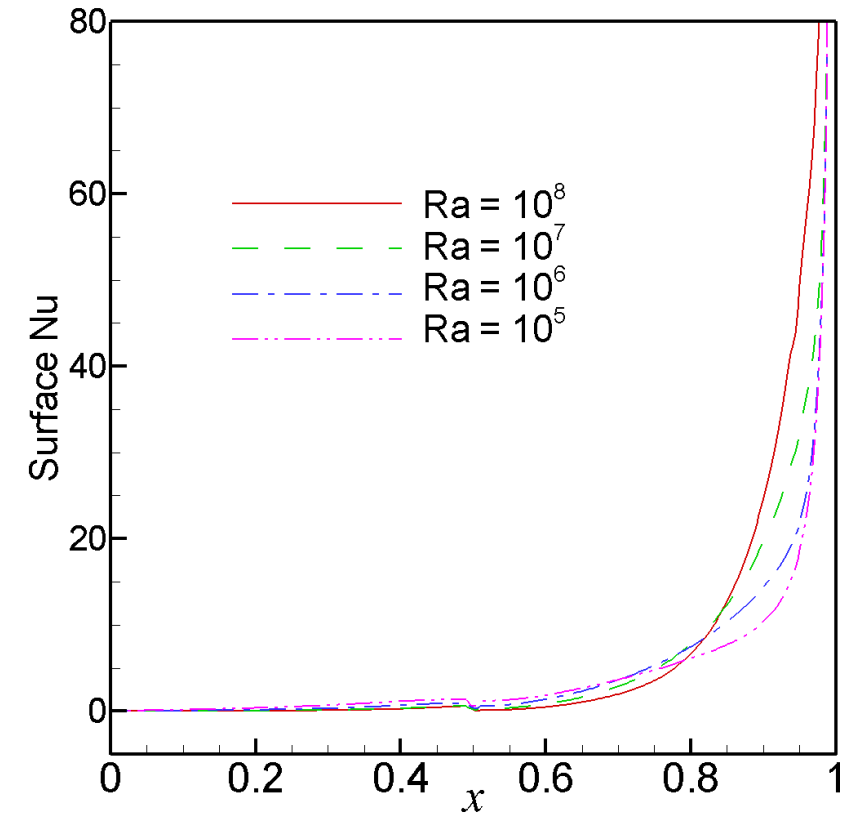

Fig 5. Surface Nu on the inclined surface of the enclosure for different Ra

\section{Conclusion}

Natural convection thermal boundary layer adjacent to the heated inclined wall of a right angled triangle with adiabatic fin mounted from that heated wall is investigated. Different $R a$ in the range of $10^{5}-10^{8}$ with fixed $A=1.0$ and $\operatorname{Pr}=0.71$ are considered in this study. The natural convective flow development is dominated by two distinct stages, i.e. a boundary-layer development stage and the thermal stratification of the cavity. It is revealed from the numerical simulations that the thermal boundary layer along the inclined surface is characterized by three distinct stages, i.e. a start-up early stage, a transitional stage and a steady stage. In presence of fin, the thermal boundary layer adjacent to the inclined wall breaks initially; then reattached with the downstream boundary layer next to the fin. Surface $N u$ increases gradually towards the tip of the heated inclined and cold bottom wall and gets its highest value at the tip due the highest temperature difference at this location. $N u$ are obtained higher for higher values of $R a$ as the natural convection becomes dominant to convection due to higher $R a$.

\section{Nomenclature}

$\begin{array}{ll}A & \text { Aspect ratio of the cavity } \\ g & \text { Acceleration due to gravity } \\ H & \text { Dimensional height of the cavity } \\ L & \text { Dimensional base length of the cavity } \\ \mathrm{Nu} & \text { Nusselt number } \\ P & \text { Dimensional pressure } \\ p & \text { Non-dimensional pressure } \\ P r & \text { Prandt number }\end{array}$

$\begin{array}{ll}R a & \text { Rayleigh number } \\ T & \text { Dimensional fluid temperature } \\ T_{\mathrm{c}} & \text { Dimensional cold temperature } \\ T_{h} & \text { Dimensional hot temperature } \\ U, V & \text { Dimensional velocity components } \\ u, v & \text { Non-dimensional velocity components } \\ X, Y & \text { Dimensional coordinates } \\ x, y & \text { Non-dimensional coordinates } \\ G r e e k & \text { symbols } \\ \beta & \text { thermal expansion coefficient } \\ \Delta T & \text { temperature difference between hot and cold } \\ & \text { wall } \\ \kappa & \text { thermal diffusivity } \\ \rho & \text { Density } \\ v & \text { kinematic viscosity } \\ \theta & \text { Non-dimensional temperature } \\ \tau & \text { Non-dimensional time }\end{array}$

\section{References}

[1] V.A. Akinsete and T.A. Coleman, "Heat transfer by steady laminar free convection in triangular enclosures". Int. J. Heat Mass Transfer, vol. 25(7) pp. 991-998, 1982.

[2] F. Moukalled and S. Acharya, "Natural convection in trapezoidal cavities with baffles mounted on the upper inclined surfaces". Numerical Heat Transfer, Part A: Applications, vol. 37 (6) pp. 545-565, 2000.

[3] F. Moukalled and S. Acharya. "Natural convection in a trapezoidal enclosure with offset baffles". J. Thermophys. Heat Transfer, vol. 15( 2), pp. 212-218, 2001.

[4] F. Xu , J. C. Patterson and C. Lei, "An experimental study of the unsteady thermal flow around a thin fin on a sidewall of a differentially heated cavity". International Journal of Heat and Fluid Flow, vol. 29 pp. 1139-1153, 2008.

[5] F. Xu , J. C. Patterson and C. Lei, "Temperature oscillations in a differentially heated cavity with and without a fin on the sidewall", International Communications in Heat and Mass Transfer, vol. 37 pp. 350-359, 2010.

[6] F. Xu , J. C. Patterson and C. Lei, "Transition to a periodic flow induced by a thin fin on the sidewall of a differentially heated cavity", International Journal of Heat and Mass Transfer,vol. 52 pp. 620-628, 2009.

[7] Y. Varol, H.F. Oztop and A. Varol, "Effects of thin fin on natural convection in porous triangular enclosures". International Journal of Thermal Sciences, vol.:46 pp. 1033-1045, 2007.

[8] E. H. Ridouane and A. Campo, "Effects of attaching baffles onto the inclined walls of attic frames for purposes of energy conservation". Heat Transfer Eng.vol. 28(2) pp. 103-111, 2007. 
[9] Y. Varol, H. F. Oztop and I. Pop, "Natural convection in porous media-filled triangular enclosure with a conducting thin fin on the hot vertical wall", Proc. IMechE. Part C: J. Mechanical Engineering Science, vol. 222(9) pp. 1735-1743, 2008 .

[10] T. N. Anderson, M. Duke and J. K. Carson, "Convection suppression in a triangular-shaped enclosure". Computational Thermal Sciences, vol. 1 pp. 309-321, 2009.
[11] T. N. Anderson, M. Duke and J. K. Carson, "Suppression of natural convection heat transfer coefficients in an attic shaped enclosure". International Communications in Heat and Mass Transfer, vol. 37 pp. 984-986, 2010.

[12] S. C. Saha, J. C. Patterson and C. Lei, "Natural convection in attics subject to instantaneous and ramp cooling boundary conditions". Energy and Buildings, vol. 42 pp.1192-1204, 2010 . 